\title{
A threshold feedback compression scheme of channel quality indicator (CQI) in LTE systems
}

\begin{abstract}
The channel quality indicator (CQI) feedback is an essential technique in describing channel state information, especially at high-speed mobility for LTE and LTE-A systems. The number of reported CQI feedback increases when a large number of users are served by eNodeB. However, this feedback inevitably entails uses and can lead to severe degradation in system throughput. Therefore, an appropriate method for reducing CQI feedback overhead must be developed. In this paper, the system throughput and bit error rate (BER) are investigated by using a threshold feedback compression scheme of CQI at low and high user speeds. Results show that the system throughput proportionally increases with the threshold values at the cost of BER. Therefore, a high threshold level is recommended under high user speed conditions to improve the tradeoff between BER and throughput. In contrast, a low threshold level is targeted to provide reliable transmission when the network is fully loaded and the user is in a bad channel state.
\end{abstract}

Keyword: CQI feedback; LTE; Threshold scheme; Best M individual; Best M average; Full CQI feedback 